\title{
EFECTO DE ZEOLITA SOBRE LA EFICIENCIA DE FERTILIZANTES NITROGENADOS EN EL CULTIVO DE ARROZ ${ }^{1}$
}

\author{
José Ezequiel Villarreal-Núñez ${ }^{2}$, Luís Alberto Barahona-Amores ${ }^{2}$, Ovidio Antonio Castillo-Ortiz ${ }^{2}$
}

\section{RESUMEN}

Efecto de zeolita sobre la eficiencia de fertilizantes nitrogenados en el cultivo de arroz. El objetivo de este estudio fue evaluar el efecto de la zeolita sobre la eficiencia de uso de fertilizantes nitrogenados en el cultivo de arroz. El experimento se estableció en El Cacao, Tonosí, provincia de Los Santos, Panamá, entre agosto y diciembre de 2012 y 2013, bajo condiciones de secano. Se utilizó la variedad de arroz IDIAP FL 106-11 con densidad de siembra de 110 $\mathrm{kg} / \mathrm{ha}$ en un Fluvisol vértico de fertilidad media. El estudio consistió de cinco tratamientos con cuatro repeticiones, un diseño de bloques completamente aleatorizados, y una dosis de $\mathrm{N}$ de $80 \mathrm{~kg} / \mathrm{ha}$, mezclado con diferentes porcentajes de zeolita $(0 ; 15 ; 25 ; 35$ y 45\%). Se empleó la técnica isotópica de ${ }^{15} \mathrm{~N} 3 \%$ en exceso, para determinar eficiencia de uso del fertilizante nitrogenado (EUFN). Cada unidad experimental consistió de $15 \mathrm{~m}^{2}$ con tres micro-parcelas de $1 \mathrm{~m}^{2}$ en donde se aplicó la urea marcada con ${ }^{15} \mathrm{~N}$ en diferentes épocas del ciclo del cultivo para determinar EUFN en cada aplicación. Los mejores rendimientos de grano se obtuvieron con $80 \mathrm{~kg}$ $\mathrm{N}+45 \%$ zeolita/ha y $80 \mathrm{~kg} \mathrm{~N}+15 \%$ zeolita/ha. Se logró un incremento en la EUFN de un 7\% con la adición de zeolita natural. Pequeñas dosis de $12 \mathrm{~kg} / \mathrm{ha}(15 \%)$ de zeolita puede mejorar la EUFN permitiendo mediante sucesivos experimentos, recomendar una disminución en la cantidad de $\mathrm{N}$ a aplicar en suelos con condiciones edafoclimáticas similares, aptos para el cultivo de arroz.

Palabras claves: técnica isotópica, agricultura de

\begin{abstract}
Effect of zeolite on the nitrogen fertilizer efficiency in rice crop. The objective of this work was to evaluate the effect of zeolite on the efficiency of nitrogen fertilizer in rice crop. The experiment was conducted in Cacao, Tonosi, Los Santos, Panama, between August and December 2012 and 2013 under rainfed conditions.The Rice variety used was IDIAP FL 106-11 with seeding density of $110 \mathrm{~kg} / \mathrm{ha}$ in a medium- fertility vertic Fluvisol. This study consisted of five treatments with four replications, following a completely randomized block design using $\mathrm{N}$ rate of $80 \mathrm{~kg} / \mathrm{ha}$ mixed with different percentages of zeolite $(0 ; 15 ; 25 ; 35$ and $45 \%$ ). The isotopic technique ${ }^{15} \mathrm{~N} 3 \%$ excess to determine the nitrogen fertilizer use efficiency (EUNF) was used. Each experimental unit consisted of $15 \mathrm{~m}^{2}$ with three micro-plots of $1 \mathrm{~m}^{2}$ where ${ }^{15} \mathrm{~N}$-labeled urea was applied at different times of the crop cycle to determine EUNF in each application. Top grain yields were obtained with $80 \mathrm{~kg} \mathrm{~N}+45 \%$ zeolite/ ha and $80 \mathrm{~kg} \mathrm{~N}+15 \%$ zeolite/ha. A $7 \%$ EUNF increase was achieved with the addition of natural zeolite. Small doses of $12 \mathrm{~kg} / \mathrm{ha}(15 \%)$ of zeolite can improve EUNF; after subsequent experiments, it is recommended to reduce the amount of $\mathrm{N}$ applied in soils with similar soil and climatic conditions suitable for growing rice.
\end{abstract}

Keywords: isotopic technique, rainfed, urea. secano, urea.

\footnotetext{
Recibido: 21 de enero, 2015. Aceptado: 17 de marzo, 2015. Proyecto financiado por el Organismo Internacional de Energía Atómica (OIEA), Viena, Austria.

2 Instituto de Investigación Agropecuaria de Panamá (IDIAP). Vía Interamericana, Divisa, provincia de Herrera, Panamá. jevilla38@gmail. com, alberline@gmail.com, ovidiocastillo@gmail.com
}

CC) (1) 2015 Agronomía Mesoamericana es desarrollada en la Universidad de Costa Rica y se encuentra licenciada con Creative
Commons Reconocimiento-NoComercial-SinObraDerivada 3.0 Costa Rica. Para más información escríbanos a pccmca@ucr.ac.cr 


\section{INTRODUCCIÓN}

El uso indiscriminado de fertilizantes sintéticos en la agricultura, sobre todo de la urea, ha tenido efectos perjudiciales en las propiedades del suelo y su conservación; se ha causado en muchos casos la acidificación de los suelos cultivables y la disminución de la materia orgánica hasta llegar a la pérdida de la capa arable, lo que ha provocado que cientos de áreas antes cultivadas quedaran en total desertificación.

Actualmente, son escasos los estudios que existen en Panamá sobre la utilización de fuentes naturales que ayuden al mejor aprovechamiento y conservación de los nutrientes en el suelo, para un mayor desarrollo de los cultivos agrícolas.

La clinoptilolita, es la zeolita más abundante en la naturaleza, posee una alta capacidad de intercambio catiónico y gran afinidad por iones amonio $\left(\mathrm{NH}_{4}^{+}\right)$ (Inglezakis et al., 2004). Su uso puede permitir un ahorro sustancial de la cantidad de fertilizante utilizado, al sustituir entre un 20 a $25 \%$ de fertilizante soluble. El aumento de la eficiencia del uso del nitrógeno, tiene gran repercusión sobre la producción vegetal y es un factor fundamental para reducir la contaminación del ambiente (Bolado et al., 2003). En trabajos previos realizados en Cuba, se determinó que al aplicar la clinoptilolita, se mejoró la eficiencia de uso de fertilizantes nitrogenados, aumentando los rendimientos y disminuyendo las dosis de nitrógeno (Loboda, 1999; Soca et al., 2004).

En Panamá, la eficiencia de uso de los fertilizantes nitrogenados en el cultivo de arroz, fluctúa entre 20 y 35\% (Name y Villarreal, 2004). Gran parte de este se pierde por volatilización del amonio, denitrificación o lixiviación de nitratos; contaminando aguas subterráneas, aguas superficiales y el ambiente.

Los productos de amplia utilización como la urea y el fosfato diamónico (DAP) (18-46-0), cuestan 0,90 y 1,14 centavos de dólar el $\mathrm{kg}$, respectivamente, mientras la zeolita tiene un valor comercial de 0,30 centavos de dólar el kilogramo (Arciniegas, comunicación personal, 2012).

Las técnicas isotópicas, utilizando fertilizantes nitrogenados marcados con el isotopo estable ${ }^{15} \mathrm{~N}$, y la técnica de dilución isotópica de ${ }^{15} \mathrm{~N}$, son fundamentales para estudiar el ciclo del nitrógeno (Urquiaga y Zapata, 2000). El uso de trazadores ${ }^{15} \mathrm{~N}$ permite monitorear el momento y la localización de un compuesto en el sistema suelo-planta-agua (Pino, 2005), y determinar la cantidad de nutriente que absorbe la planta y la que se pierde en el ambiente.

En Panamá no se habían estudiado los beneficios producidos por la zeolita para mejorar la absorción de nitrógeno por los cultivos; por lo que el objetivo de este estudio fue evaluar el efecto de la zeolita sobre la eficiencia de uso de fertilizantes nitrogenados en el cultivo de arroz.

\section{MATERIALES Y MÉTODOS}

El experimento se realizó en el corregimiento El Cacao, distrito de Tonosí, provincia de Los Santos, ubicado a los $7^{\circ} 26^{\prime} 03^{\prime \prime}$ de latitud norte y $80^{\circ} 24^{\prime} 37^{\prime \prime}$ de longitud oeste, durante los años 2012 y 2013, bajo condiciones de secano, en un suelo Fluvisol vértico de fertilidad media (Proyecto Zonificación de Suelos de Panamá), entre los meses de agosto y diciembre de cada año.

Se utilizó la variedad de arroz IDIAP FL 106-11, por su buena adaptabilidad al sistema edafoclimático de la región. Se utilizó una densidad de siembra de $110 \mathrm{~kg} / \mathrm{ha}$.

El suelo presentó una textura tipo franco con un $\mathrm{pH}$ adecuado para la liberación de la mayor parte de los nutrientes del suelo; contiene un nivel elevado de fósforo, calcio y magnesio, comparado con otros suelos de Panamá y valores medios de potasio y materia orgánica (Cuadro 1).

El experimento consistió de cinco tratamientos con cuatro repeticiones cada uno (Cuadro 2), en un diseño estadístico de bloques completos al azar, para minimizar el efecto de la variabilidad espacial del suelo. Cada unidad experimental (UE) consistió de quince hileras separadas a $0,20 \mathrm{~m}(3 \mathrm{~m})$ con $5 \mathrm{~m}$ de largo $\left(15 \mathrm{~m}^{2}\right)$; con una separación de $0,50 \mathrm{~cm}$ entre parcelas y $1 \mathrm{~m}$ entre los bloques. En cada UE se utilizaron tres micro-parcelas de $1 \mathrm{~m}^{2}$ cada una, donde se aplicó el isótopo estable ${ }^{15} \mathrm{~N}$.

Se utilizó super fosfato triple (0-46-0) y cloruro de potasio 0-0-60 $(\mathrm{KCl})$ como principal fuente de $\mathrm{P}_{2} \mathrm{O}_{5}$ y $\mathrm{K}_{2} \mathrm{O}$; respectivamente, y urea $46 \% \mathrm{~N}$ como fuente de $\mathrm{N}$ para el abono base.

El fertilizante nitrogenado fue fraccionado en tres aplicaciones y tres épocas distintas: a la siembra, a los 30 días después de la siembra (dds) y a los 55 dds. Lo anterior se realizó con el fin de poder determinar la 
Cuadro 1. Análisis de suelo, antes de establecer el ensayo, de la región de Cacao, Tonosí, provincia de Los Santos, Panamá. 2012. Table 1. Soil analysis, before starting the experiment, in the Region of Cacao, Tonosi, Los Santos province, Panama. 2012.

\begin{tabular}{|c|c|c|c|c|c|c|c|c|c|c|c|}
\hline $\begin{array}{c}\text { Ar-L- } \\
\text { Arc }\end{array}$ & pH & $\mathbf{P}$ & $\mathbf{K}$ & $\mathbf{C a}$ & Mg & Al & MO & Mn & $\mathbf{F e}$ & Zn & $\mathrm{Cu}$ \\
\hline$\%$ & $1: 2,5$ & \multicolumn{2}{|c|}{$\mathrm{mg} / \mathrm{kg}$} & \multicolumn{3}{|c|}{-----cmol(+)/kg---- } & $\%$ & \multicolumn{4}{|c|}{-------------mg/kg----------- } \\
\hline $38-42-20$ & 6,1 & 31 & 121 & 31 & 7,4 & 0,1 & 2,2 & 67 & 60 & 1,5 & 4,5 \\
\hline
\end{tabular}

Ar: arena (sand); L: limo (silt); Arc: arcilla (clay); MO: materia orgánica (organic matter).

Cuadro 2. Tratamientos empleados en los experimentos utilizando dosis de zeolita como un porcentaje de la dosis de nitrógeno aplicada. Cacao, Tonosí, provincia de Los Santos, Panamá. 2012-2013.

Table 2. Treatments used in the experiment using doses of zeolite as a percentage of the dose of nitrogen applied. Cacao, Tonosi, Los Santos province, Panama. 2012-2013.

\begin{tabular}{ccc}
\hline \multirow{2}{*}{ Tratamiento } & Nitrógeno & Zeolita \\
\cline { 2 - 3 } & $\mathbf{( k g / h a )}$ & $(\mathbf{\%})$ \\
\hline 1 & 80 & 0 \\
2 & 80 & 15 \\
3 & 80 & 25 \\
4 & 80 & 35 \\
5 & 80 & 45 \\
\hline
\end{tabular}

eficiencia en la extracción de $\mathrm{N}$ por el cultivo en las diferentes aplicaciones del fertilizante. Se aplicó urea marcada con ${ }^{15} \mathrm{~N}$ en el fertilizante de base, el cual se colocó en la primera micro-parcela (30\%), fertilizando el resto de la UE con urea sin marcar. A los 30 dds, se realizó la segunda aplicación de urea marcada en el $40 \%$ de la segunda micro-parcela y urea normal en el resto. Finalmente, a los 55 dds se realizó la tercera aplicación de urea marcada en el 30\% de la tercera micro-parcela y normal en el resto de la UE.

Se calculó el contenido de $\mathrm{N}$ total y de urea ${ }^{15} \mathrm{~N}$ en follaje, grano y suelo; producción de biomasa del cultivo, rendimiento de grano, porcentaje de $\mathrm{N}$ proveniente del fertilizante (Nddf), porcentaje de eficiencia de uso del fertilizante nitrogenado (EUFN), análisis de varianza, comparación de medias por medio de la prueba de Duncan, correlación entre dosis de zeolita y EUFN.

La Figura 1, muestra la precipitación total observada en la región de Cacao, Tonosí, durante el ciclo del cultivo en el año 2013.

\section{RESULTADOS Y DISCUSIÓN}

Para la variable producción de biomasa (Cuadro 3), se encontró que la mayor producción se obtuvo con los tratamientos $80 \mathrm{~kg} \mathrm{~N} / \mathrm{ha}+35 \%$ y $80 \mathrm{~kg} \mathrm{~N} / \mathrm{ha}+45 \%$ de zeolita. Los otros tratamientos $80 \mathrm{kgN} / \mathrm{ha}+15 \%$ y 80 $\mathrm{kgN} / \mathrm{ha}+25 \%$ de zeolita no presentaron diferencia con el testigo ni con los tratamientos ya mencionados.

En lo referente a la variable rendimiento de grano (Cuadro 3), hubo una tendencia a observar mayores rendimientos al mezclar $80 \mathrm{~kg}$ de N/ha con $45 \%$ y $80 \mathrm{~kg}$ de N/ha con $15 \%$ de zeolita. Sin embargo, no hubo diferencia estadísticamente significativa con el testigo ni tampoco con los otros tratamientos. Este resultado pudo haber sido muy influenciado por el comportamiento errático de las precipitaciones durante el desarrollo del experimento, ya que estas fueron muy irregulares y estuvieron por debajo del nivel mínimo requerido en la zona de estudio, lo que afectó el rendimiento. Por su parte, Ulloa (2006) en suelos de Tumbes, Perú, tampoco encontró diferencias significativas en rendimiento de grano y biomasa al combinar diferentes dosis de fertilizantes nitrogenados con zeolita.

Cuando se observó el resultado total para la microparcela 1 (Cuadro 4), donde se aplicó el 30\% de la dosis del fertilizante nitrogenado a la siembra, se encontró que el Nddf y la EUFN total en follaje y grano, fueron mayores en el tratamiento $80 \mathrm{~kg} \mathrm{~N} / \mathrm{ha}+$ $15 \%$ de zeolita, presentando una eficiencia de uso del fertilizante de 11,52\%, aprovechando $2,77 \mathrm{~kg} \mathrm{~N} / \mathrm{ha}$ de un total de $24 \mathrm{~kg} \mathrm{~N} / \mathrm{ha}$ aplicados.

Para el total de la microparcela 2 (Cuadro 5), se encontró que el tratamiento $80 \mathrm{~kg} \mathrm{~N} / \mathrm{ha}+15 \%$ de zeolita fue el que mostró los mejores resultados; sin embargo, no se diferenció estadísticamente del testigo. Este tratamiento tuvo una EUFN de 34,25\% 


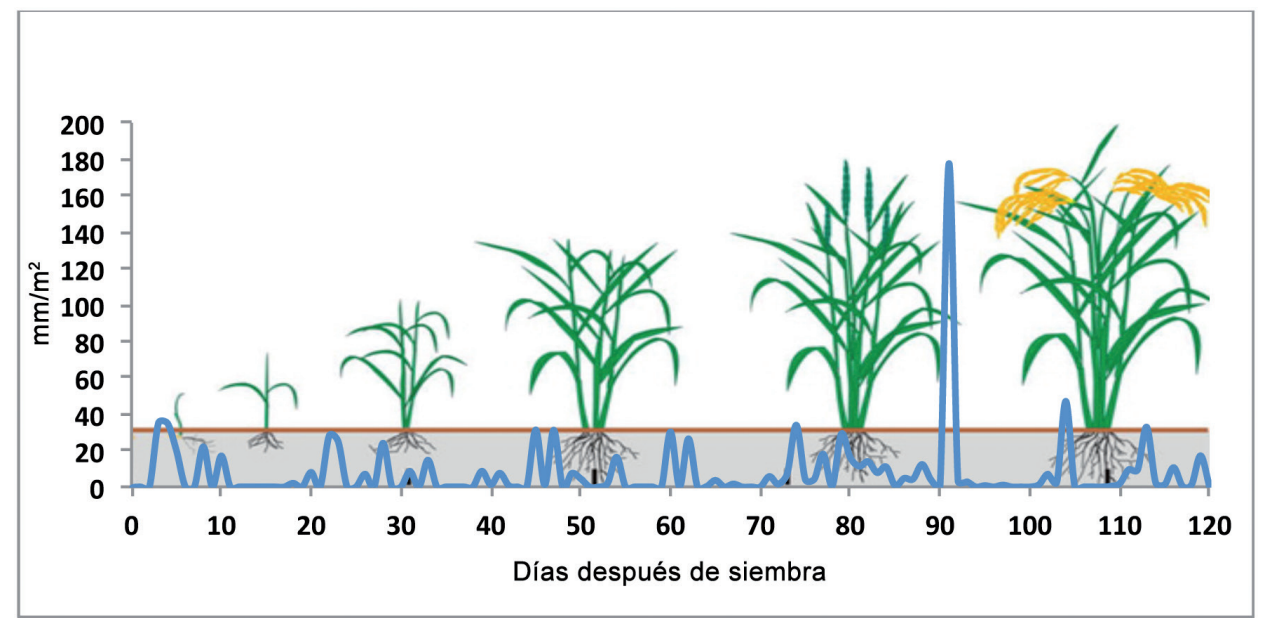

Figura 1. Precipitación durante el desarrollo del ensayo, realizado bajo condiciones de secano. Cacao, Tonosí, provincia de Los Santos, Panamá. Agosto-diciembre, 2013.

Figure 1. Precipitation during the experiment development, conducted under rainfed conditions. Cacao, Tonosi, Los Santos Province, Panama. August - december, 2013.

Cuadro 3. Promedio de rendimiento de biomasa y grano en los experimentos realizados con el cultivo de arroz, bajo condiciones de secano, en Cacao, Tonosí, provincia de Los Santos, Panamá. 2012-2013.

Table 3. Average of biomass and grain yield in experiments with rice crop, under rainfed conditions, in Cacao, Tonosi, Los Santos province, Panama. 2012-2013.

\begin{tabular}{cccccc}
\hline \multicolumn{2}{l}{ Tratamientos } & \multicolumn{2}{c}{$\begin{array}{c}\text { Rendimiento de } \\
\text { Biomasa* }\end{array}$} & $\begin{array}{c}\text { Rendimiento de } \\
\text { Grano* }\end{array}$ \\
\hline $\mathbf{N}$ & + & Zeolita & $------------k g / h a-----------$ \\
kg/ha & $\%$ & & & \\
\hline 80 & + & 0 & 8522,10 & $\mathrm{~B}$ & $3547,25 \mathrm{AB}$ \\
80 & + & 15 & $10018,32 \mathrm{AB}$ & $3697,08 \mathrm{~A}$ \\
80 & + & 25 & $9282,93 \mathrm{AB}$ & $3576,04 \mathrm{AB}$ \\
80 & + & 35 & $12428,04 \mathrm{~A}$ & $3366,89 \mathrm{AB}$ \\
80 & + & 45 & $12348,14 \mathrm{~A}$ & $3874,02 \mathrm{~A}$ \\
\hline
\end{tabular}

*Significativo a nivel $\geq 0,05$ de probabilidad / Significant at $\geq$ 0,05 probability.

Medias seguidas de la misma letra no presentan diferencia estadísticamente significativa / Means followed by the same letter do not show, statistically significant difference.

lo que indica que se aprovecharon 10,96 kg de N/ha de un total de $32 \mathrm{~kg}$ de N/ha aplicados. Este resultado muestra que con solo aplicar el $15 \%$ de zeolita en relación a la cantidad de $\mathrm{N}$ aplicada ya se puede
Cuadro 4. Nitrógeno total en el follaje y grano del cultivo de arroz proveniente del fertilizante (Nddf total) y eficiencia de uso del fertilizante nitrogenado (EUFN) aplicado a la siembra en la microparcela 1 . Cacao, Tonosí, provincia de Los Santos, Panamá. 2012-2013.

Table 4. Total nitrogen from fertilizer (Nff) on the foliage and grain of rice and nitrogen fertilizer use efficiency (EUNF) applied to planting in microplot 1. Cacao, Tonosi, Los Santos province, Panama. 2012-2013.

\begin{tabular}{|c|c|c|c|c|}
\hline \multicolumn{3}{|c|}{ Tratamientos } & \multirow[b]{2}{*}{$\begin{array}{c}\text { Nddf total } \\
\text { kg/ha }\end{array}$} & \multirow[b]{2}{*}{$\begin{array}{c}\text { EUFN*2 } \\
\%\end{array}$} \\
\hline $\begin{array}{c}\text { N } \\
\text { kg/ha }\end{array}$ & + & $\begin{array}{c}\text { Zeolita } \\
\%\end{array}$ & & \\
\hline 80 & + & 0 & 1,90 & 7,94 В \\
\hline 80 & + & 15 & 2,77 & $11,52 \mathrm{~A}$ \\
\hline 80 & + & 25 & 2,06 & $8,57 \quad \mathrm{~B}$ \\
\hline 80 & + & 35 & 1,78 & $7,43 \quad$ В \\
\hline 80 & + & 45 & 2,10 & 8,74 B \\
\hline
\end{tabular}

*Significativo a nivel $\geq 0,05$ de probabilidad / Significant at $\geq$ 0.05 probability.

Medias seguidas de la misma letra no presentan diferencia estadísticamente significativa / Means followed by the same letter do not show statistically significant difference.

${ }^{1}$ Nitrógeno proveniente del fertilizante / nitrogen from fertilizer. 2 Eficiencia de uso del fertilizante nitrogenado / nitrogen fertilizer use efficiency (EUNF). 
Cuadro 5. Nitrógeno total en el follaje y grano del cultivo de arroz proveniente del fertilizante y eficiencia de uso del fertilizante nitrogenado aplicado 30 días después de la siembra en la microparcela 2. Cacao, Tonosí, provincia de Los Santos, Panamá. 20122013.

Table 5. Total nitrogen from fertilizer on the foliage and grain of rice and nitrogen fertilizer use efficiency applied 30 days before planting in microplot 2 . Cacao, Tonosi, Los Santos province, Panama. 2012-2013.

\begin{tabular}{|c|c|c|c|c|}
\hline \multicolumn{3}{|c|}{ Tratamientos } & \multirow[b]{2}{*}{$\begin{array}{c}\text { Nddf total }^{1} \\
\text { kg/ha }\end{array}$} & \multirow[b]{2}{*}{$\begin{array}{c}\text { EUFN }^{* 2} \\
\%\end{array}$} \\
\hline $\begin{array}{c}\mathrm{N} \\
\mathrm{kg} / \mathrm{ha}\end{array}$ & + & $\begin{array}{c}\text { Zeolita } \\
\%\end{array}$ & & \\
\hline 80 & + & 0 & 9,36 & $29,34 \mathrm{AB}$ \\
\hline 80 & + & 15 & 10,96 & $34,25 \mathrm{~A}$ \\
\hline 80 & + & 25 & 6,93 & $21,66 \mathrm{C}$ \\
\hline 80 & + & 35 & 9,77 & $30,55 \mathrm{AB}$ \\
\hline 80 & + & 45 & 9,83 & $30,71 \mathrm{AB}$ \\
\hline
\end{tabular}

$*$ Significativo a nivel $\geq 0,05$ de probabilidad / Significant at $\geq$ 0,05 probability.

Medias seguidas de la misma letra no presentan diferencia estadísticamente significativa / Means followed by the same letter do not show statistically significant difference.

${ }^{1}$ Nitrógeno proveniente del fertilizante / nitrogen from fertilizer.

2 Eficiencia de uso del fertilizante nitrogenado / nitrogen fertilizer use efficiency (EUNF).

mejorar la absorción del $\mathrm{N}$ desde las primeras etapas del cultivo.

En la microparcela 3 se encontró que todos los tratamientos fueron estadísticamente similares entre sí y superiores al testigo, a excepción del $80 \mathrm{~kg} \mathrm{~N} / \mathrm{ha}+$ $15 \%$ de zeolita que no fue diferente estadísticamente al testigo (Cuadro 6). La EUFN fue mayor al $80 \%$, lo que indica que se aprovecharon más de $19 \mathrm{~kg}$ de N/ ha de un total de $24 \mathrm{~kg}$ de $\mathrm{N}$ aplicados a los 55 días después de la siembra. En suelos ferralíticos de Cuba, también encontraron resultados similares al obtenido cuando combinaron urea con $15 \%$ de zeolita (Muñiz et al., 1999).

Al comparar la EUFN de la parcela completa (incluye las tres microparelas, Cuadro 7), todos los tratamientos fueron similares y mostraron diferencia estadística con el testigo. En números absolutos fue
Cuadro 6. Nitrógeno total en el follaje y grano del cultivo de arroz proveniente del fertilizante y eficiencia de uso del fertilizante nitrogenado aplicado a los 55 días después de la siembra en la microparcela 3. Cacao, Tonosí, provincia de Los Santos, Panamá. 2012-2013.

Table 6. Total nitrogen from fertilizer on the foliage and grain of rice and nitrogen fertilizer use efficiency applied 55 days before planting in microplot 3 . Cacao, Tonosi, Los Santos province, Panama. 2012-2013.

\begin{tabular}{ccccc}
\hline \multicolumn{3}{c}{ Tratamientos } & & \\
\cline { 1 - 1 } $\begin{array}{c}\mathbf{N} \\
\text { Kg/ha }\end{array}$ & $\begin{array}{c}\text { Zeolita } \\
\text { \% }\end{array}$ & $\begin{array}{c}\text { Nddf total } \\
\text { kg/ha }\end{array}$ & $\begin{array}{c}\text { EUFN } \\
\text { \%2 }\end{array}$ \\
\hline 80 & + & 0 & 15,42 & $64,24 \mathrm{~B}$ \\
80 & + & 15 & 17,38 & $72,42 \mathrm{AB}$ \\
80 & + & 25 & 20,05 & $83,55 \mathrm{~A}$ \\
80 & + & 35 & 19,43 & $80,95 \mathrm{~A}$ \\
80 & + & 45 & 19,80 & $82,50 \mathrm{~A}$ \\
\hline
\end{tabular}

**Significativo a nivel $\geq 0,05$ de probabilidad / Significant at $\geq$ 0,05 probability.

Medias seguidas de la misma letra no presentan diferencia estadísticamente significativa / Means followed by the same letter do not show statistically significant difference.

${ }^{1}$ Nitrógeno proveniente del fertilizante / nitrogen from fertilizer.

2 Eficiencia de uso del fertilizante nitrogenado / nitrogen fertilizer use efficiency (EUNF).

superior el tratamiento $80 \mathrm{~kg} \mathrm{~N} / \mathrm{ha}+45 \%$ de zeolita $(40,65 \%)$ lo que significa que se aprovecharon $32,52 \mathrm{~kg}$ de N/ha de la dosis de $80 \mathrm{~kg} \mathrm{~N} / \mathrm{ha}$ aplicados al cultivo, mejorando en un $7 \%$ la EUFN.

En la Figura 2 se muestra la correlación entre el porcentaje de zeolita aplicado y la EUFN; en ella se observa una cierta estabilización luego de aplicar 12\% de zeolita al suelo; teniendo en cuenta que la aplicación fue de $80 \mathrm{~kg}$ de N/ha, el 12\% correspondería a unos 9,6 $\mathrm{kg}$ de zeolita/ha.

La ecuación polinomial que muestra un $\mathrm{R}^{2}$ de $82,7 \%$, indica que con una aplicación de $10 \mathrm{~kg}$ de zeolita/ha ya se tendría una EUFN arriba de $38 \%$, suficiente para el desarrollo del cultivo. Al no existir diferencias estadísticas para rendimiento de grano, se podría decir que aplicando $10 \mathrm{~kg}$ de zeolita y $70 \mathrm{~kg}$ de $\mathrm{N} /$ ha se tendría una absorción de $\mathrm{N}$ total suficiente 
Cuadro 7. Nitrógeno total en follaje y granos del cultivo de arroz proveniente del fertilizante y eficiencia de uso del fertilizante nitrogenado por tratamiento. Parcela completa. Tonosí, provincia de Los Santos, Panamá. 2012-2013.

Table 7. Total nitrogen from fertilizer on the foliage and grain of rice and nitrogen fertilizer use efficiency per treatment. Whole plot. Tonosi, Los Santos province, Panama. 2012-2013.

\begin{tabular}{ccccc}
\hline \multicolumn{3}{c}{ Tratamientos } & & \\
\cline { 1 - 1 } $\begin{array}{c}\mathbf{N} \\
\text { Kg/ha }\end{array}$ & $\begin{array}{c}\text { Zeolita } \\
\text { \% }\end{array}$ & $\begin{array}{c}\text { Nddf total } \\
\text { \% }\end{array}$ & $\begin{array}{c}\text { EUFN*2 } \\
\text { kg/ha }\end{array}$ \\
\hline 80 & + & 0 & $25,17 \mathrm{~B}$ & $33,81 \mathrm{~B}$ \\
80 & + & 15 & $31,52 \mathrm{~A}$ & $39,40 \mathrm{~A}$ \\
80 & + & 25 & $30,34 \mathrm{~A}$ & $37,93 \mathrm{~A}$ \\
80 & + & 35 & $31,71 \mathrm{~A}$ & $39,64 \mathrm{~A}$ \\
80 & + & 45 & $32,52 \mathrm{~A}$ & $40,65 \mathrm{~A}$ \\
\hline
\end{tabular}

*Significativo a nivel $\geq 0,05$ de probabilidad / Significant at $\geq$ 0,05 probability.

Medias seguidas de la misma letra no presentan diferencia estadísticamente significativa / Means followed by the same letter do not show statistically significant difference.

${ }^{1}$ Nitrógeno proveniente del fertilizante / nitrogen from fertilizer.

2 Eficiencia de uso del fertilizante nitrogenado / nitrogen fertilizer use efficiency (EUNF).

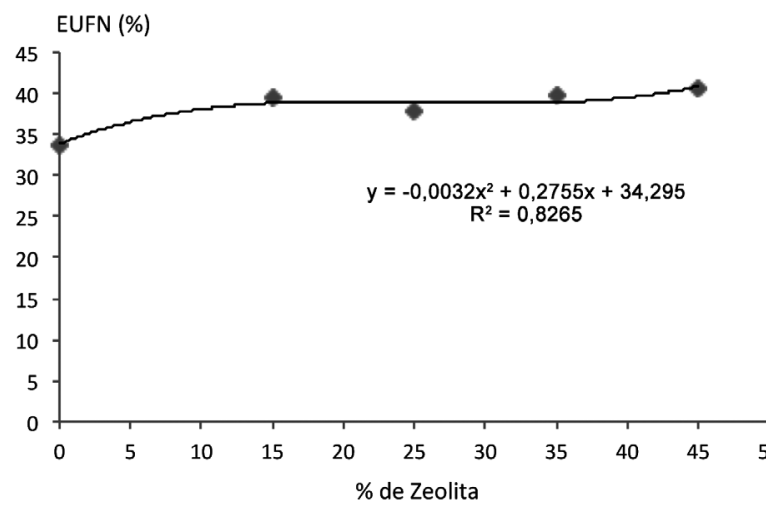

Figura 2. Correlación entre porcentaje de zeolita aplicado y eficiencia de uso del fertilizante nitrogenado en un cultivo de arroz sembrado bajo condiciones de secano. Cacao, Tonosí, provincia de Los Santos, Panamá. 2012-2013.

Figure 2. Correlation between the percentage of zeolite applied and nitrogen fertilizer use efficiency on rice seed culture under rainfed conditions. Cacao, Tonosi, Los Santos province, Panama. 2012-2013. para el cultivo de arroz, ya que el testigo absorbió $27 \mathrm{~kg}$ de N/ha. Lo anterior significa una disminución de $10 \mathrm{~kg}$ de $\mathrm{N} / \mathrm{ha}(12,5 \%$ de $\mathrm{N})$ para el cultivo y consecuentemente ahorro para el productor.

Al realizar un ensayo con el cultivo de maíz en Guerrero, México, González et al. (2012) encontraron que al sustituir con clinoptilolita el $15 \%$ en peso de la fórmula 90-60-00, no incrementaron significativamente el rendimiento de grano, la altura ni el grosor de las plantas, sin embargo, mejoró en un $10 \%$ la absorción de N.

Respecto a lo que se retuvo de fertilizante nitrogenado en el suelo, mediante la técnica del análisis del isotopo ${ }^{15} \mathrm{~N}$ aplicado en exceso, se pudo comprobar, al comparar su contenido en los tratamientos $80 \mathrm{~N}-0 \%$ zeolita y $80 \mathrm{~N}-25 \%$ zeolita en las profundidades de $0-$ $20 \mathrm{~cm} ; 20-40 \mathrm{~cm}$ y $40-60 \mathrm{~cm}$, que la zeolita no tuvo mayor influencia en la misma, ya que en ambos tratamientos se retuvo casi el $36 \%$ de la dosis de ${ }^{15} \mathrm{~N}$ aplicada.

A pesar de que en los dos años en que se realizó el experimento no hubo diferencia significativa en rendimiento de grano entre el testigo y los tratamientos, la adición del mineral demostró que con pequeñas dosis (apenas 12\%) de zeolita se puede mantener y mejorar la eficiencia de uso del fertilizante nitrogenado permitiendo disminuir las cantidades de $\mathrm{N}$ a aplicar.

La técnica del uso de isotopos estables como el ${ }^{15} \mathrm{~N}$, demostró ser efectiva para comprobar la eficiencia de uso del fertilizante nitrogenado aplicado, cuánto fue absorbido por la planta y cuánto permaneció en el suelo.

\section{LITERATURA CITADA}

Bolado, S.A., M. Alonso, y J. Alvarez-Benedi. 2003. Caracterización de procesos acoplados de adsorción, transformación y volatilización de $\mathrm{N}$ en suelos fertilizados con urea. En: J. Alvarez-Benedi, y R. Marinero, editores, Estudios de la zona no saturada del suelo. Volum. VI. Instituto Tecnológico Agrario de Castilla y León (ITA), Valladolid, ESP. p. 185-192.

González, M., N.O. Gómez, J. Muíz, F. Valencia, D. Gutiérrez, y H. Figueroa. 2012. Rendimiento del maíz de riego tratado con zeolita más fertilizantes químicos en el estado de Guerrero. Rev. Mex. Cienc. Agríc. 3(6):1129-1144.

Inglezakis, V.M., P. Loizidou, and H. Grigoropoulou. 2004. Ion exchange studies on natural and modified zeolites and the concept of exchange site accessibility. Soil Sc. Soc. Amer. J. 62:622-629.

Agron. Mesoam. 26(2):315-321. 2015 ISSN 2215-3608 
Loboda, B. 1999. Agroecological assessment of using substrates from zeolite containing rock in greenhouse grown sweet pepper. Agrokhimiya 2:67-72.

Muñiz, O., G. Dueñas, A. Nuviola, M. Biart, R. Beltran, C. John, T. Sanchez, and F. Alvarez. 1999. Use of isotope techniques in order to evaluate the efficiency of chemical fertilizers in rice. In: Proceedings of II International Symposium on Nuclear Agriculture, Industry and Environment. Porto Alegre, Brasil, 18 to 21 april 1999. Ed. Génesis, EMBRAPA, Rio Grande do Sul, BRA. p. 83.

Name, B., y J.E. Villarreal. 2004. Compendio de resultados de investigación del programa de suelos del IDIAP. IDIAP, PAN.

Pino, I. 2005. Técnicas isotópicas en estudios de suelos. http://mazinger.sisib.UChile.cl/repositorio/lb/ ciencias_agronomicas/miscelaneasagronomicas38/ C15.html (consultado 30 mayo 2012).
Soca, M., J. Castellanos, y J. Febles. 2004. Efecto de la zeolita en la eficiencia de los fertilizantes químicos, fertilizantes y enmiendas de origen mineral. Panorama Minero 14:261-268.

Ulloa, V. 2006. Efecto de la zeolita en la fertilización nitrogenada en el cultivo de Oryza sativa L. (arroz) var. IR43 en siembra a trasplante en Tumbes. Tesis Ing. Agr., Universidad Nacional de Tumbes, Corrales, Tumbes, PER.

Urquiaga, D., y F. Zapata. 2000. Metodologías isotópicas para estudios de la eficiencia de la fertilización nitrogenada y otros procesos del ciclo del N. En: S. Urquiaga, y F. Zapata, editores, Manejo eficiente de la fertilización nitrogenada de cultivos anuales en America Latina y el Caribe. Génesis, Embrapa, Porto Alegre, BRA. p. 25-29. 
